\title{
Heat distribution over normal and abnormal joints: thermal pattern and quantification
}

\author{
RICHARDS. SALISBURY, GRAHAM PARR, MALCOLM DE SILVA, \\ BRIAN L. HAZLEMAN, AND D. P. PAGE-THOMAS
}

From the Department of Rheumatology, Addenbrooke's Hospital, Hills Road, Cambridge

SUMMARY We have identified regular thermal patterns over normal knee, ankle, and elbow joints and demonstrate how synovitis affecting these joints may be identified by alteration or loss of the thermal pattern. Sixty healthy volunteers were thermographed on a total of 190 occasions, and 614 out of 618 joints conformed to the normal thermal pattern. Eight-five patients with synovitis of at least one of the specified joints were thermographed on a total of 339 occasions, and 322 out of 1362 thermograms were abnormal. No joint with clinical evidence of synovitis had a normal thermal pattern. As temperature-based parameters have been found to show marked diurnal variation and relative frequency distributions do not have this drawback, we suggest that quantification of synovitis by thermography should in future be based on abnormalities of thermal pattern rather than absolute skin temperature values.

The objective assessment of disease activity in rheumatoid arthritis is difficult. Many parameters are based on patients' symptoms, which may not give an accurate indication of the progress of the disease, and laboratory evaluation can be unhelpful. ${ }^{1}$ Infrared thermography (IRT) has been used for the past 10 years to measure skin temperature over inflamed joints. $^{2}{ }^{3}$ Horvath and Hollander ${ }^{4}$ measured the intra-articular temperature in patients with rheumatoid arthritis and noted that it could be used as a guide to the acuteness of inflammation. Bacon $e t$ $a l .{ }^{5}$ showed that measurement of mean skin temperature could be used as a measure of disease activity. Skin temperature is affected by many internal and external factors. ${ }^{6}$ Circadian rhythm, metabolic rate, calorific intake, physical activity, emotional state, and atmospheric temperature and humidity can all exert a marked effect on skin temperature. In particular, owing to the proximity of blood vessels to the skin in the extremities even small alterations in vascular tone will produce large changes in skin temperature.

Many of these factors are uncontrollable. As a result there may be considerable variation in a single joint at different times of measurement. If measurements of absolute skin temperature are made, this variation may lead to the suggestion of inflammation where none is present and vice versa.

Accepted for publication 28 July 1982.

Correspondence to Dr B. Hazleman, Rheumatology Research Unit, Addenbrooke's Hospital, Hills Road, Cambridge CB2 $2 Q Q$.
In addition, the variation leads to difficulties in standardisation and relating a single measurement to an arbitrary 'normal' temperature.?

We therefore set out to examine whether such relation to absolute arbitrary standards is necessary and whether inspection of the thermogram could identify the presence or absence of synovitis. Thermograms of the knee, elbow, and ankle joints of normal subjects have been compared with those from patients with clinical evidence of synovitis. To test the effect of diurnal variation on the methods of analysis serial readings were obtained at different times of day.

\section{Methods and patients}

Thermography. This was carried out in a draught-free room with ambient temperature controlled to $20.5^{\circ} \mathrm{C}$ $\pm 0.5^{\circ} \mathrm{C}$ and humidity $50 \% \pm 10 \%$. Subjects were seated in a modified dentist's chair with their limbs exposed and a sleeveless jacket worn.

An AGA Thermovision 680 medical system was used to detect infrared emission, and all thermograms were recorded in digital form on magnetic tape for subsequent analysis with a Varian 620 computer. The camera was placed parallel (vertical and horizontal planes) and at a distance of $1.0 \mathrm{~m}$ to thermograph elbows and ankles and $1.2 \mathrm{~m}$ for knee joints. Knees and ankles were flexed to $90^{\circ}$ and ankles to $45^{\circ}$. Thermograms were recorded of the lateral aspect of the joints. In all instances recordings were made after 
a 15-minute equilibration period in the room. Thermography was carried out between 9 am and 12 noon and between $2 \mathrm{pm}$ and $5 \mathrm{pm}$ (Table 1).

Thermogram interpretation. We define the normal thermal pattern over a joint as one in which there is a negative temperature gradient from the centre to the periphery of the joint without localised areas of increased temperature. In general this gradient is of the order of $2-3^{\circ} \mathrm{C}$ (Fig. 1).

The thermographic appearance of synovitis is characterised by loss of the negative temperature gradient or by a localised area of increased tempera-

Table 1 Number of subjects thermographed at each time of day*

\begin{tabular}{llll}
\hline & \multicolumn{2}{l}{ Number of subjects thermographed } \\
\cline { 2 - 4 } & Morning & Afternoon & $\begin{array}{l}\text { Morning and } \\
\text { afternoon }\end{array}$ \\
\hline Controls (60) & $52(100)$ & $46(93)$ & $43(80)$ \\
Patients (85) & $57(186)$ & $48(97)$ & $25(54)$ \\
\hline
\end{tabular}

*Number of occasions in brackets. ture (Fig. 2). Thermograms were classified in a single blind manner without knowledge of a subject's group or clinical details.

Quantification of relative frequency distributions of thermographs. A region of interest on the thermogram is chosen to correspond to a fixed area of surface anatomy over the joint. The analogue thermal signal from the AGA $680 \mathrm{M}$ signal is digitised into 256 grey levels by an A/D converter. A count is then made of the number of picture points which occur at each grey level. Finally, each number is divided by the total number of picture points in the area of interest giving a relative frequency distribution (RFD). Fig. 3 shows a 'normal' and 'arthritic' type of RFD. As the disease process remits, serial studies show a gradual transition from the widely spread irregular position of the arthritic towards a progressively more narrow and symmetrical leptokurtic distribution, as is usually found in normal joints.

In an initial attempt to quantify these changes we have fitteda Gaussian curve to the major components of the distribution (neglecting all values $<15 \%$ of the modal frequency). Such fitted curves approximate
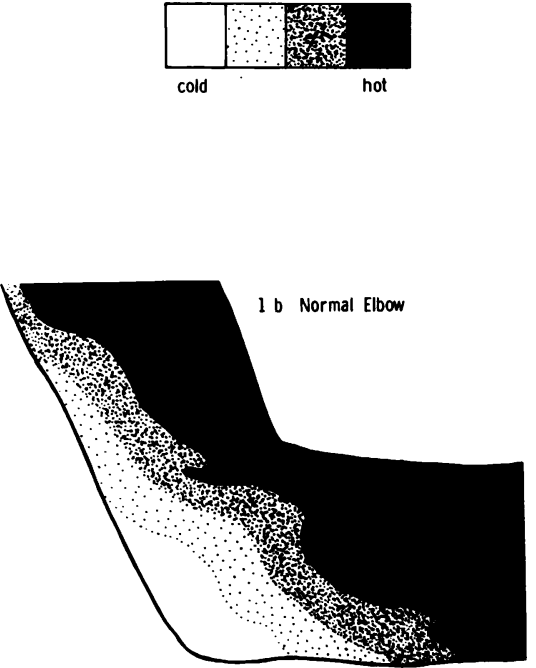
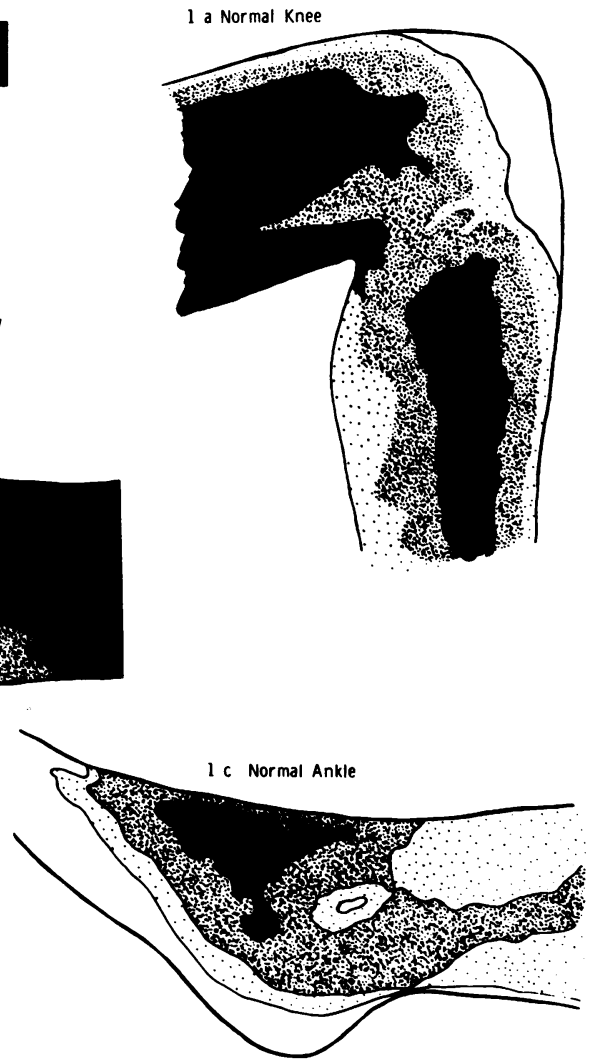

Fig. 1 Distribution of heat over (a) normal knee joint, (b) normal elbow joint, (c) normal ankle joint. 
2 a Knee with diffuse abnormality

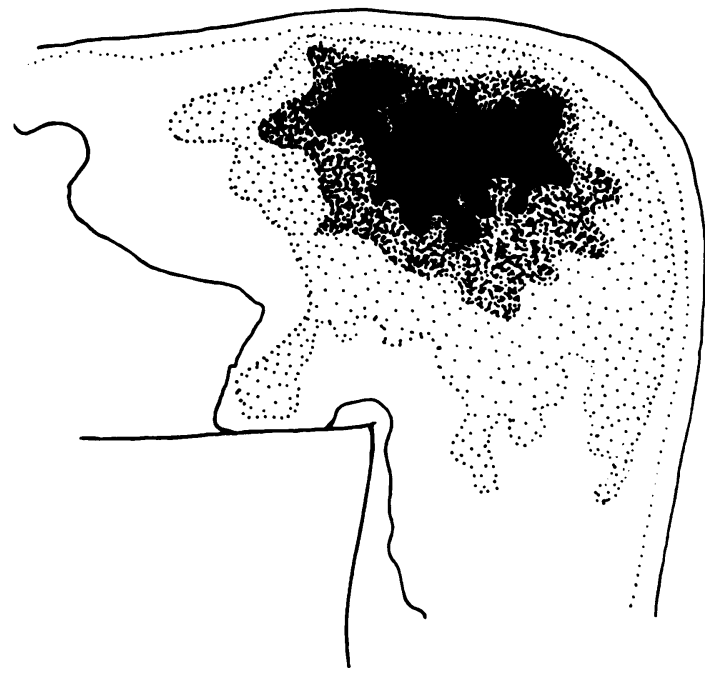

2 b Localised abnormality

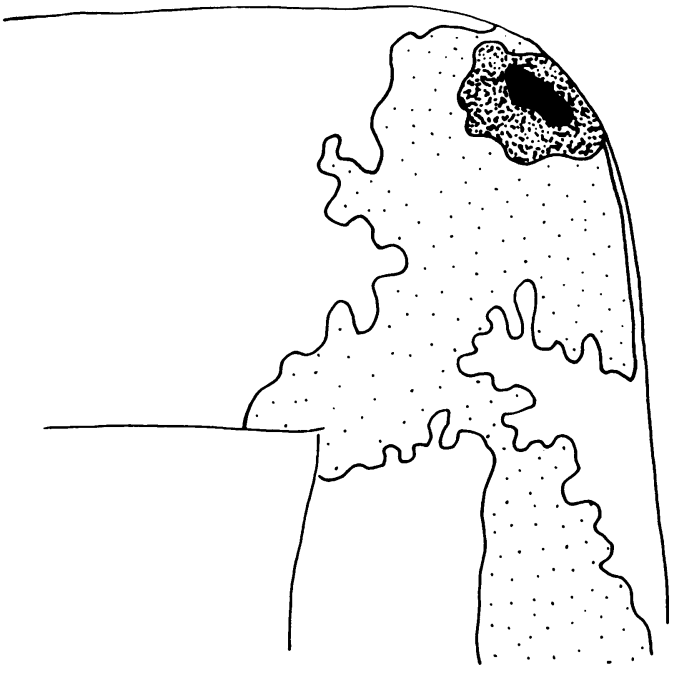

Fig. 2 Distribution of heat over knee with (a) generalised abnormal pattern, and (b) focal abnormal pattern.

very closely to the width of the 'shoulders' of both normal and abnormal distributions, though of course they do not follow closely the irregular frequency plateau found in actively inflamed joints. A calculation of the width of the RFD is made as \pm 1 SD from the mean frequency = heat distribution index (HDI) or $2 \mathrm{~S}$ value.

The HDI as an approximation to the RFD curves has been found to work well in arthritic patients. In
Relative Frequency Distribution

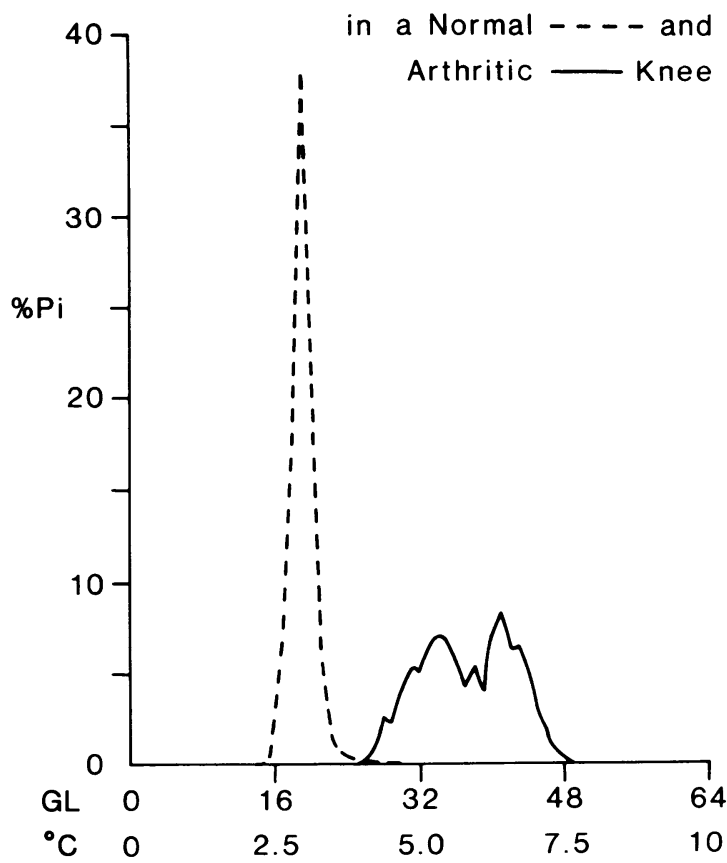

Fig. 3 Relative frequency distribution in a normal and arthritic knee. $G L=$ grey level. $C=$ Celsius equivalent. $\% P i=$ percentage proportion of grey levels.

the normal and control groups, however, some individuals were found in whom there were excessively acute 'normal' gradients, i.e., there were approximately twice the number of parallel running isotherms going from the joint centre to the periphery. Because the HDI is only a one-dimensional representation of the heat image, that is, it has no spatial relationships, it may give a spuriously high numerical value in such normal individuals.

In order to compare values for the HDI and thermographic index (TI) the knee joints of 50 patients with rheumatoid arthritis were thermographed at different times of day over a 12-month period. Inflammation in the knee joint was graded clinically by an independent observer on a unit scale $(0-3)$ corresponding to none, mild, moderate, and severe.

Knee joint values were matched according to their clinical assessment group $(1,2,3)$. Patients were thermographed both in the morning (1000-1200 hours) and afternoon (1400-1600 hours).

Control group. The control group was composed of 60 healthy volunteers drawn from the staff of Cambridge Area Health Authority, local firms, and industry. Subjects with a history of inflammatory or 
Table 2 Number of individual joints thermographed*

\begin{tabular}{lllll}
\hline Subjects & \multicolumn{4}{l}{ Number of joints thermographed } \\
\cline { 2 - 5 } & Knees & Ankles & Elbows & Total \\
\hline Controls (60) & $368(4)$ & $112(0)$ & $138(0)$ & $618(4)$ \\
Patients (85) & $588(322)$ & $380(40)$ & $394(56)$ & $1362(322)$ \\
\hline
\end{tabular}

*Number of abnormal thermograms in brackets.

symptomatic joint disease were excluded from the study. Thirty men and 30 women with ages ranging between 13 and 79 (mean 41.5 years) were thermographed on at least 2 occasions (mean 2.85). Over a 12 -month period 190 separate visits were made and a total of 618 joints were thermographed (Table 2).

Patient group. Patients were referred for study from the Rheumatology Department, Addenbrooke's Hospital. Eighty-five patients (35 males and 50 females) with ages ranging between 15 and 75 (mean 46.6 years) were thermographed on at least 3 occasions (mean 3.99). Over a 12-month period 339 separate visits were made and a total of 1362 joints were thermographed (Table 2). Patients were admitted to the study if at the time of referral there was symptomatic and clinical evidence of synovitis in at least one of the joints that were studied (knees, ankles, and elbows). Eighty-two patients had definite or 'classical' rheumatoid arthritis (American Rheumatism Association classification), 2 patients had ankylosing spondylitis with peripheral joint involvement (knee), and 1 patient had Behçet's syndrome.

\section{Results}

A characteristic 'normal' thermographic appearance of the knee joints, ankles, and elbows was found in 59 out of 60 control subjects: 614 out of 618 joints thermographed conformed to a normal pattern. In one subject a localised area of increased temperature in the right knee was consistently found on 4 occasions. Three hundred and twenty-two 'abnormal' joint thermograms were found in 85 patients (Table 2 ). Of these abnormal thermograms 319 correspond with joints showing clinical synovitis. In 2 of the remaining 3 joints which had abnormal thermograms but were clinically normal, synovitis developed within 10 days of thermography.

No normal thermograms were found of joints with clinical and symptomatic evidence of synovitis, but 39 normal thermograms were recorded of joints which were painful alone. The presence or absence of a normal thermal pattern was not dependent on the time of day that thermography was carried out.

The HDI reflects the pattern and spread of temperature over a joint, and this correlated well (Table 3)
Table 3 Significance of HDI values for assessment group at both times of day: significance of differences between the clinical subgroups $1,2,3$

\begin{tabular}{|c|c|c|c|c|c|c|c|}
\hline \multicolumn{4}{|c|}{ am Comparison } & \multicolumn{4}{|c|}{ pm Comparison } \\
\hline \multicolumn{2}{|c|}{ Group 1} & \multirow[t]{2}{*}{2} & \multirow[t]{2}{*}{3} & \multicolumn{2}{|c|}{ Group 1} & \multirow[t]{2}{*}{2} & \multirow[t]{2}{*}{3} \\
\hline 1 & - & & & 1 & - & & \\
\hline 2 & 0.001 & - & & 2 & 0.001 & - & \\
\hline 3 & 0.001 & 0.001 & - & 3 & 0.001 & 0.001 & - \\
\hline
\end{tabular}

Table 4 Significance of TI values for assessment groups at both times of day: significance of differences between the clinical subgroups $1,2,3$

\begin{tabular}{|c|c|c|c|c|c|c|c|}
\hline \multicolumn{4}{|c|}{ am Comparison } & \multicolumn{4}{|c|}{ pm Comparison } \\
\hline Group & 1 & 2 & 3 & Group & 1 & 2 & 3 \\
\hline 1 & - & & & 1 & - & & \\
\hline 2 & 0.001 & - & & 2 & NS & - & \\
\hline 3 & 0.001 & NS & - & 3 & 0.01 & NS & - \\
\hline
\end{tabular}
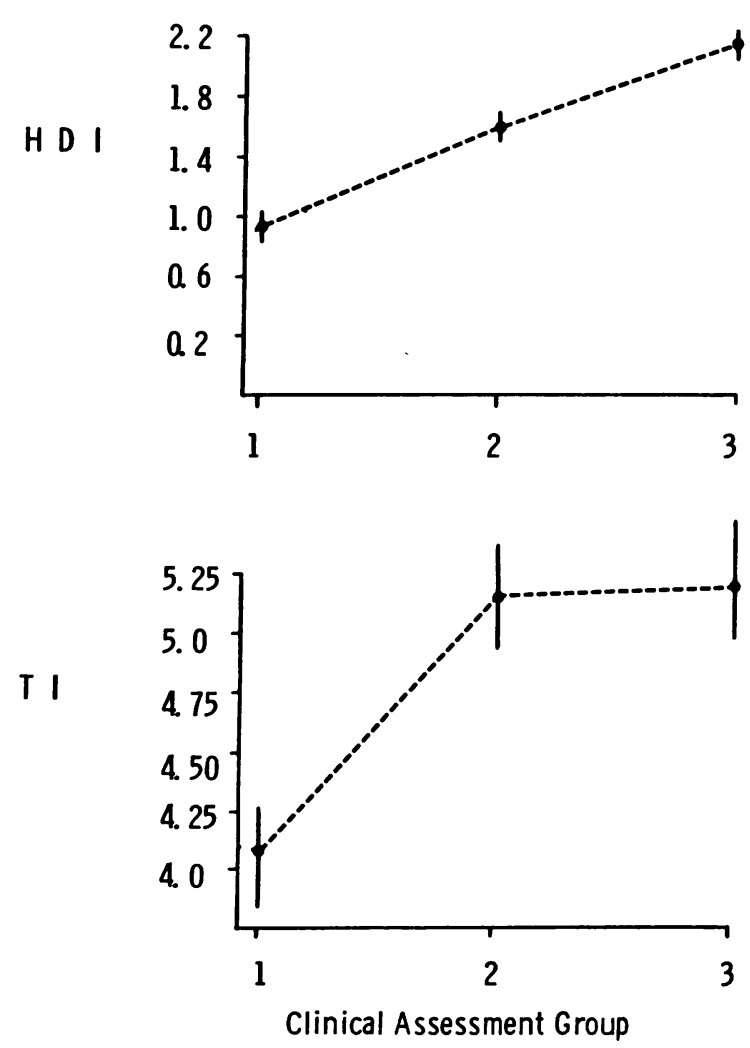

Fig. 4 Comparison of heat distribution index and thermographic index with clinical assessment (am). 

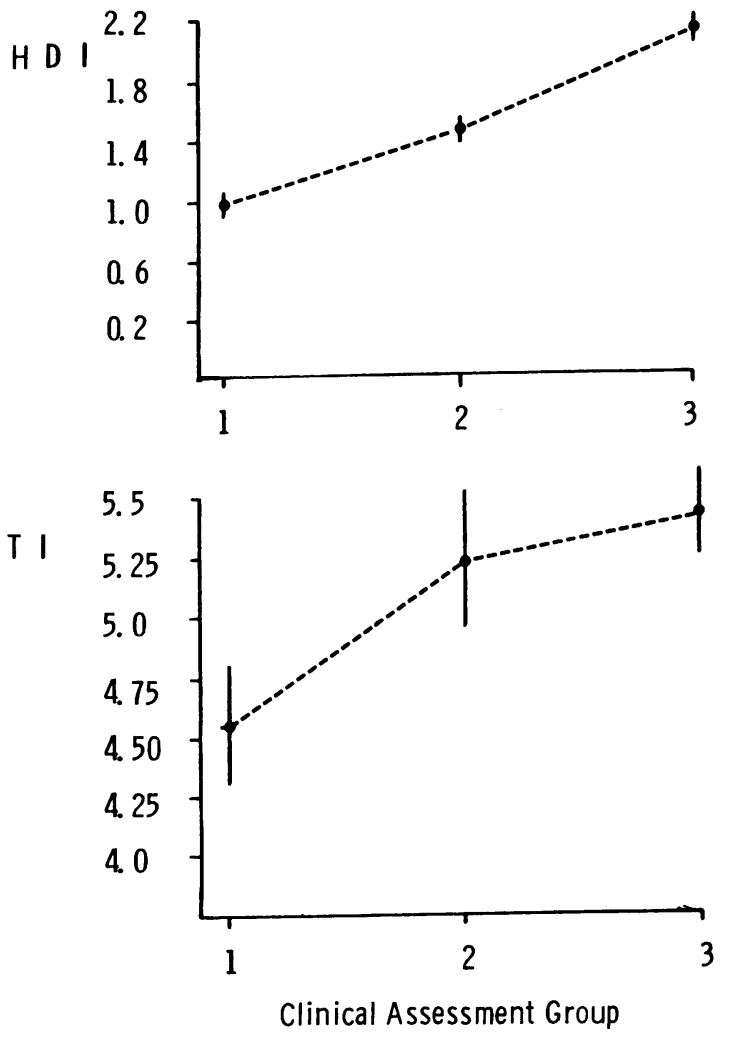

Fig. 5 Comparison of heat distribution index and thermographic index with clinical assessment (pm).

with the clinical assessment of the severity of inflammation. The TI, reflecting mean surface temperature, shows less significance of difference between individual assessment groups, particularly in the afternoon (Table 4).

There is an approximately linear relationship between HDI and the clinical assessment both in the morning (Fig. 4) and in the afternoon (Fig. 5). There is considerable overlap for TI values, particularly in the afternoon, as shown by the large standard errors. This results in significance only between individual assessment groups 1 (moderate) and 2 (severe) for TI values.

\section{Discussion}

There is still a need for an objective, reproducible, accurate method of assessing inflammatory joint disease. Thermography is a noninvasive method which appeared to satisfy these criteria and has been used to measure the mean surface temperature over a joint. However, difficulties in maintaining a stable environment and the physiological alterations of skin temperature have limited the usefulness of the technique. ${ }^{78}$ Radiometry similarly has been used successfully and cheaply, although the same limitations apply. ${ }^{910}$ Thermography differs from radiometry in providing a pictorial display of the heat distribution.

Several authors have stressed the importance of establishing normal thermal patterns, ${ }^{11}{ }^{12}$ but these have proved to be elusive..$^{13}{ }^{14}$ Throughout the literature, however, mention is made of 'abnormal thermograms' and of 'hot spots', but without prior definition of the normal. ${ }^{12}{ }^{15}$ The identification of normal patterns of heat distribution over joints and their distinctive alteration with inflammation, which we now report, allows alternative methods of quantification based on pattern of heat distribution. Our results show that in the absence of synovitis a normal thermal pattern can be identified in the knee, ankle, wrist, and elbow joints. The presence of synovitis may be repeatedly identified, and this is not affected by the time of day. In the 39 joints that were symptomatic without clinical evidence of synovitis and with a normal thermogram, it is possible that the symptoms were due to mechanical factors within the joint rather than to synovial inflammation. One control subject had an abnormal thermogram of her right knee on the 4 occasions she was seen, but there was no clinical or symptomatic evidence of inflammation.

The peripheral joints are relatively avascular and superficial. A negative temperature gradient exists from the centre of a normal joint to the skin. The heat generated by synovial inflammation will distort this gradient, and the normal thermal pattern is lost. By cooling the skin at an ambient temperature of $20^{\circ} \mathrm{C}$, the sensitivity of surface temperature gradients to subcutaneous blood flow will be increased. ${ }^{16}$ These patterns are thermal representations of underlying metabolism and blood supply. Although skin temperature may show considerable fluctuation depending upon blood flow, the distribution of heat, being more related to structure, appears relatively constant.

Several authors ${ }^{317}$ have described the thermogram of a normal knee as having a cold area corresponding to the patella in the anterior view. We have observed this also in many knees affected by synovitis. The patella may act as a heat shield, and we recommend a lateral view of the joint which minimises the problem. Love ${ }^{18}$ suggests that most alterations in surface heat distribution are due to alterations in vascular flow rather than simply due to increased metabolism, and the work of Niellson $e t$ $a l .{ }^{19}$ supports this. The loss of the negative thermal gradient seen in the inflamed knee may well be due to increased vascularity of the inflamed synovial membrane and the shunting of blood from the deep veins to the cutaneous circulation. 
The TI correlated well with clinical assessment of disease activity in several rigidly controlled studies. ${ }^{20-22}$ However, skin temperature in the normal subject is particularly affected by circadian rhythm and metabolic rate. ${ }^{23}$ This results in considerable overlap between temperature values for normal and inflamed joints, although there are distinct differences in their heat pattern. The diurnal variations in surface temperature values tend to be less conspicuous with increasing degrees of inflammation. This is not surprising, since severely inflamed joints tend to approach normal core temperature values.

Physiological variation in temperature of normal and the less inflamed joints may account for the poor correlation found between thermography and other methods of assessment in some studies. In this study we found a considerable overlap between skin temperature (TI) over normal and abnormal knees, particularly in the afternoon for all but the most inflamed joints.

The identification of normal and abnormal patterns of heat distribution over the surface of a joint allows alternative methods to distinguish and quantify inflammation. We have used a simple statistic to measure the range of heat distribution for a joint and have found that it correlated well with clinical assessment of disease activity. The results of HDI measurement confirm the observation that under standardised environmental conditions the pattern of heat distribution over a joint as expressed by the relative frequency distribution of grey levels remains relatively constant during the day, although the absolute temperature value may change. Thus the heat distribution patterns of a single joint may be moved up or down a temperature range depending on the individual's physiological or environmental changes, while the actual thermal spread of the heat pattern remains constant. These diurnal/circadian changes in surface temperature illustrate the inaccuracies in methods of assessment of inflammation based on absolute skin temperature, while the relative constancy of these heat patterns confirms the usefulness of quantification based on the relative temperature distribution.

This work has been supported by a grant from the Arthritis and Rheumatism Council.

We thank Dr E. P. Wraight and the staff of the Nuclear Medicine Department for their help and use of their facilities.

\section{References}

1 Beecher H K. Measurement of subjective responses. Oxford: Oxford University Press, 1959.
2 Haberman J D, Ehrlich G E, Levenson C. Thermography in rheumatic diseases. Arch Phys Med Rehabil 1968; 49: 187-92.

3 Tiselius P. Studies on joint temperature, joint stiffness and muscle weakness in rheumatoid arthritis. Acta Rheumatol Scand 1969; suppl 14.

4 Horvath S M, Hollander J L. Intra-articular temperature as a measure of joint reaction. J Clin Invest 1949; 28: 469-73.

5 Bacon P A, Collins A J, Ring F J, Cosh J A. Thermography in the assessment of inflammatory arthritis. Clin Rheum Dis 1976; 2: 51-65.

6 Reinberg A. Circadian changes in the temperature of human beings. Clin Rheum Dis 1976; 2: 128-39.

7 Huskisson E G, Berry H, Browett J P, Wykenham Balme H. Measurement of inflammation. II. Comparison of technetium clearance and thermography with standard methods in a clinical trial. Ann Rheum Dis 1973; 32: 99-102.

8 Grayson M E. Knee joint temperatures during the use of antiinflammatory drugs. Ann Clin Res 1974; 6: 167-70.

9 Ring E F J, Cosh J A. Skin temperature measurement by radiometry. Br Med $J$ 1968; iv: 448.

10 Bird H A, Calguneri M, Leatham P A, Wright V. Measurement of temperature in the arthritic hand. Rheumatol Rehabil 1980; 19: 205-11.

11 Brueschke E, Haas C. Image analysis of medical thermograms. Invest Radiol 1969; 4: 28-33.

12 Branemark P I, Ekholm R, Goldie I, Lindstrom J. Synovectomy in rheumatoid arthritis. Acta Rheumatol Scand 1967; 13: 161-89.

13 Brueschke E, Haberman-Brueschke J D, Gershon-Cohen J. Infra-red thermoprofile analysis in clinical medicine. Am J Med Electr 1965; 4: 65-9.

14 Cosh J A, Ring E F J. Thermography and rheumatology. Rheumatol Rehabil 1970; 10: 342-8.

15 Haberman J D, Ehrlich G E, Levenson C. Thermography in rheumatic diseases. Arch Phys Med 1968; 49: 187-92.

16 Stolwijk J A J. In: Uematsu S, ed. Medical thermography: theory and clinical applications. Los Angeles: Brentwood Publishing, 1976: 40-51.

17 Collins A J, Ring E F J, Bacon P A, Brookshaw J D. Thermography and radiology. Complementary methods for the study of inflammatory diseases. Clin Radiol 1976; 27: 237-43.

18 Love $\mathrm{T} \mathrm{J}$. Thermography as an indicator of blood perfusion. Thermal characteristics of tumours. Applications in detection and treatment. Ann NY Acad Sci 1980; 335: 416-28.

19 Nielsson S K, Gustafsson S E, Torell L M. Skin temperature over a heat source: experimental studies and theoretical calculations. Thermal characteristics of tumours. Applications in detection and treatment. Ann NY Acad Sci 1980; 335: 416-28.

20 Bacon P A , Collins A J, Cosh J A. Thermographic assessment of the anti-inflammatory effect of flurbiprofen in rheumatoid arthritis. Curr Med Res Opin 1975; 3: suppl 4; $20-6$.

21 Bacon P A, Collins A J, Ring E F J, Cosh J A. Thermography in the assessment of inflammatory arthritis. Clin Rheum Dis 1976; 2: 51-65.

22 Bacon P A, Ring E F J, Collins A J. Thermography in the assessment of antirheumatic agents. In: Gordon $\mathbf{J} L$, Hazelman B L, eds. Rheumatoid arthritis. Elsevier/North Holland, Biomedical Press, Amsterdam: 1977: 105-10.

23 Conroy R T W L, Mills J N. Temperature: human circadian rhythms. London: Churchill, 1970: 18-26. 